\title{
Effect of Astaxanthin, Vitamin E, and Vitamin C in Combination with Calorie Restriction on Sperm Quality and Quantity in Male Rats
}

\author{
Aliasghar Vahidinia, Ali Reza Rahbar \& Mohammad Mehdi Shakoori \\ Mahmoodabadi
}

To cite this article: Aliasghar Vahidinia, Ali Reza Rahbar \& Mohammad Mehdi Shakoori Mahmoodabadi (2016): Effect of Astaxanthin, Vitamin E, and Vitamin C in Combination with Calorie Restriction on Sperm Quality and Quantity in Male Rats, Journal of Dietary Supplements, DOI: 10.1080/19390211.2016.1211783

To link to this article: http://dx.doi.org/10.1080/19390211.2016.1211783

曲 Published online: 02 Aug 2016.

Submit your article to this journal $₫$

Q View related articles $\asymp$

View Crossmark data $₫$ 


\title{
Effect of Astaxanthin, Vitamin E, and Vitamin C in Combination with Calorie Restriction on Sperm Quality and Quantity in Male Rats
}

\author{
Aliasghar Vahidinia ${ }^{1}$, Ali Reza Rahbar ${ }^{2}$, \\ \& Mohammad Mehdi Shakoori Mahmoodabadi ${ }^{1}$ \\ ${ }^{I}$ Department of Nutrition, School of Medicine, Hamadan University of Medical \\ Sciences, Hamadan, I.R. Iran, ${ }^{2}$ Department of Nutrition, The Persian Gulf Tropical \\ Medicine Research Center, Bushehr University of Medical Sciences, Bushehr, I.R. Iran
}

\begin{abstract}
The aim of this study was to investigate the effect of calorie restriction and dietary antioxidant supplementation separately or in combination on the quality and quantity of sperm in male rat. Forty male rats were randomly allocated to four groups of 10 animals each, and fed for at least 86 days with an ad libitum diet (group 1), a restricted diet (group 2), an ad libitum diet and astaxanthin, vitamin E, and vitamin C supplements (group 3), or a restricted diet with astaxanthin, vitamin E, and vitamin C supplements (group 4). At the end of the study period, sperm count and motility were determined with a hemocytometer, and differences between the groups were analyzed by analysis of variance. In addition, total antioxidant capacity and 8-epi prostaglandin F2 alpha were measured at the beginning and end of the study period with an enzymelinked immunosorbent assay method. After 86 days, a significantly higher sperm count was seen in group 4 compared to other groups. The percentage of immotile sperm was significantly decreased in groups 2,3 , and 4 in comparison to group 1 . A significant increase in total antioxidant capacity was observed in group $3(p=0.02)$ and group 4 $(p=0.02)$ compared to groups 1 and 2 . Antioxidant supplementation with or without calorie restriction had no significant effect on serum isoprostane level in any group. Astaxanthinin, combined with vitamin $\mathrm{E}$, vitamin $\mathrm{C}$, and calorie restriction, was able to ameliorate, in part, infertility in male rats.
\end{abstract}

KEYWORDS. astaxanthin, (8-epi-PGF2 $\alpha$ ), 8-epi prostaglandin F2 alpha, infertility, male, total antioxidant capacity, vitamin $\mathrm{C}$, vitamin $\mathrm{E}$

\section{INTRODUCTION}

Infertility in humans is the lack of conception after one year of intercourse (Stephen \& Chandra, 2006), and the rate of infertility in men is believed to be about 25 to $30 \%$ (Katib, 2015). The quantity and quality of sperm in men with

Address correspondence to: Ali Reza Rahbar, Department of Nutrition, The Persian Gulf Tropical Medicine Research Center, Bushehr University of Medical Sciences, Bushehr, I.R. Iran. (E-mail: rahbar_alireza@ yahoo.com; rahbar@bpums.ic.ir) 
infertility is reduced concurrently with changes in lifestyle. Obesity is one of the changes that can lead to infertility (Jensen et al., 2004; Katib, 2015), and in this connection, it was reported that overweight and obesity in men are linked to reduced testosterone levels (Sallmen et al., 2006). It is reported that glycation end products cause sperms dysfunction (Vlassara \& Palace, 2002). Calorie restriction is known to improve insulin resistance and reduce the amount of advanced glycation end products (Aris et al., 2010). However, in some experimental studies, calorie restriction has failed to potentiate fertility (Roth et al., 2000); therefore, other factors apparently play an essential role in infertility.

Oxidative stress, a pathological situation that may be related to unsuccessful reproductive activity (Palmer et al., 2012; Zorn, Vidmar, \& Meden-Vrtovec, 2003) has been a source of concern as a primary cause of male infertility (Vijayprasad, Ghongane, \& Nayak, 2014). Lipid peroxidation is a major event in oxidative stress (du Plessis et al., 2011). The testicular membranes contain large amounts of polyunsaturated fatty acids, and are thus consequently at risk of peroxidation (Vijayprasad et al., 2014). In experimental cryptorchidism, the rate of antioxidant enzyme activity is decreased, and this situation is associated with augmented lipid peroxidation (Vijayprasad et al., 2014).

Reportedly, lipid peroxidation in the testes makes this tissue vulnerable to oxidative stress (Aitken \& Roman, 2008). In this connection, it should be noted that spermatogenesis is a tremendously dynamic replicative process that produces approximately 1,000 new sperms per second in humans (Aitken \& Roman, 2008). The speed of cell division in this system thus requires high rates of oxygen consumption by mitochondria in the germinal epithelium (Aitken \& Roman, 2008). Under stressful conditions, spermatozoa create low amounts of reactive oxygen species (ROS). However, excessive ROS produced by leukocytes and immature sperms during rapid sperm proliferation unambiguously damage sperm deoxyribonucleic acid (DNA) (Agarwal \& Saleh, 2002). In this connection, Agarwal, Tvrda, and Sharma (2014) demonstrated significantly elevated levels of ROS in teratozoospermia, and Gvozdjakova et al. (2015) reported enhanced sperm density after supplementation with ROS-scavenging antioxidants such as L-carnitine, fumarate, ubiquinol, vitamin $\mathrm{E}$, and vitamin $\mathrm{C}$.

Astaxanthin, a red-orange xanthophyll carotenoid extracted from algae, has a number of clinical benefits and is high in antioxidant activity (Wang et al., 2015). This carotenoid has a greater antioxidant activity than other carotenoids because of the hydroxyl and ketonic end groups located on the ionone rings of its chemical structure (Guerin, Huntley, \& Olaizola, 2003). Reportedly, astaxanthin improves human sperm capacitation by increasing its activation (Andrisani et al., 2015). However, astaxanthin is highly susceptible to oxygen, light, and auto-oxidation (Vasantha, Rupasinghe, \& Yasmin, 2010). In addition, there are several studies that show no change in ROS content following astaxanthin addition in human cells (Pashkow, Watumull, \& Campbell, 2008), in which astaxanthin did not directly scavenge superoxides. This is probably because astaxanthin, being extremely lipophilic, can be inserted in the bilayer, while the charged radicals are unable to cross cell membranes so that contact with astaxanthin becomes improbable or impossible (Wolf et al., 2010). Several studies have shown that $\alpha$-tocopherol and ascorbic acid are less reactive with lipid radicals than astaxanthin, and can 
interact with oxidized forms of astaxanthin to regenerate astaxanthin in a timecourse reaction (Sigurgisladottir et al., 1994; Zhang \& Omaye, 2001). However, the impact of antioxidant supplementation on sperm integrity and motility is small and controversial (Katib, 2015).

We, therefore, designed the present study to investigate the effect of antioxidant supplementation separately and in combination with calorie restriction on the quality and quantity of sperm in male rats. A mixture of astaxanthin, vitamin $\mathrm{E}$, and vitamin $\mathrm{C}$ was used to prevent pro-oxidation processes and favor astaxanthin regeneration (Vincent et al., 2006). To control for the influence of dietary fat on total antioxidant capacity (TAC) (Nagata et al., 2000), total fat intake was held constant during the study period.

\section{MATERIAL AND METHODS}

Forty specific pathogen-free young male Wistar rats, 30 days old and weighing 180-250 g, were provided by the Medical Center Animal Research Facility at Hamedan University of Medical Sciences. During the study period they were housed in micro-filter-top cages in the animal facility at the Nutrition Department Laboratory of Hamedan University of Medical Sciences. The animals were allowed to acclimatize for one week at $22.3^{\circ} \mathrm{C}$ with a $12: 12$-h light:dark cycle and $60-65 \%$ humidity, and were provided with rodent chow and water ad libitum. The rat diet was supplied by Sahand-Dastchin Co. (Tabriz, Iran) and included the following to cover the animals' nutritional requirements: brown rice $10.2 \%$, oats $2.2 \%$, wheat $4.6 \%$, soy $21.752 \%$, fish meal $2.82 \%$, calcium carbonate $0.711 \%$, dehydrated yeast culture $1.5 \%$, flax seed meal (linseed) $4.5 \%$, inulin $5.153 \%$, monocalcium phosphate $1.68 \%$, soy oil $35 \%$, cellulose $6.46 \%$, potassium citrate $2.132 \%$, cysteine $0.388 \%$, choline chloride $0.25 \%$, vitamins $1.292 \%$, and minerals $1.292 \%$. The animals were randomly allocated to four groups of 10 animals each, which were fed with an ad libitum diet (group 1); a restricted diet (group 2); an ad libitum diet combined with astaxanthin, vitamin E, and vitamin $\mathrm{C}$ supplements (group 3); and a restricted diet combined with astaxanthin, vitamin $\mathrm{E}$, and vitamin $\mathrm{C}$ supplements (group 4), for at least 86 days. Rats in group 3 (ad libitum diet and astaxanthin, vitamin E, and vitamin C supplements) and group 4 (restricted diet with astaxanthin, vitamin E, and vitamin $\mathrm{C}$ supplements) were gavaged every day at 9 am with astaxanthin, vitamin $\mathrm{E}$, and vitamin $\mathrm{C}$ in corresponding doses of 100,100 , and $200 \mathrm{mg} / \mathrm{kg}$, respectively. The rats in all groups continued to consume their usual diet while taking the antioxidant mixture. Antioxidants (vitamin E, vitamin C, and astaxanthin) were supplied by Zahravi Co. (Tehran, Iran).

Before the study, the amount of food consumed by the rats ad libitum was measured, and $30 \%$ less was given to animals in groups 2 and 4 as the restricted diet. All animals were weighed daily throughout the study period, and the amount of food consumed was measured. At the beginning and end of the study period, blood samples were taken from the tail to measure TAC and 8-epi prostaglandin F2 alpha (8-epi-PGF2 $\alpha$ ) with an enzyme-linked immunosorbent assay (ELISA) method. At the end of the 86th day of the study, the rats were given a lethal overdose of isoflorane by inhalation and killed. 
The cauda epididymis was quickly detached and the supporting fat, blood vessels, and connective tissue were separated and positioned on a plate. The organs were washed with normal saline to remove blood. Then the cauda epididymis was incised longitudinally with scissors and compressed with forceps to obtain sperms, which were placed in a Petri dish with phosphate buffered saline.

\section{SPERM COUNT ANALYSIS}

Sperm count was done with a hemocytometer under a light microscope. The caudal epididymal sperm solution $(10 \mu \mathrm{L})$ was loaded in a hemocytometer and covered with a cover slip. The hemocytometer was placed under a light microscope and viewed under $\times 400$ magnification. Sperm count was recorded by counting $4 \times 4$ squares horizontally or vertically.

Final sperm count was determined with the following formula described previously by Rathje, Johnson, and Lunstra (1995):

Sperm count $=$ total number of sperms in 5 squares $\times 50,000$

$$
\times 100(\text { cells } / \mathrm{mL}) \text {. }
$$

Only sperm heads that were entirely within the squares were counted.

\section{SPERM MOTILITY ANALYSIS}

A hemocytometer was used for sperm motility analysis. After $10-\mu \mathrm{L}$ caudal epididymal sperm solution was loaded and covered with a cover slip, the hemocytometer was placed under a light microscope and viewed under $\times 400$ magnification. The light from the microscope was kept dim to reduce the effect of heat on sperm motility. The motility grade of spermatids was recorded when they entered the $4 \times 4$ squares horizontally or vertically (Table 1 ). Before statistical analysis, the raw data were tabulated as percentages with the following formula:

Percentage of sperms in a given grade $=$ Number of sperms in that grade $\times 100 /$ Total number of sperms in all grades.

\section{ANTIOXIDANT EVALUATION}

Plasma TAC was determined with the new-generation 2,2'-azinobis-(3ethylbenzothiazoline-6-sulfonic acid) radical cation method (Randox, Kearneysville, WV, USA) (Erel, 2004). Serum 8-epi prostaglandin F2 $\alpha$ was

TABLE 1. Sperm motility characteristics

\begin{tabular}{ll}
\hline Grade & Characteristics \\
Grade 1 & Immotile sperm \\
Grade 2 & Sperm with non-progressive motility: No forward movement despite vibration-like \\
& tail movements \\
Grade 3 & Sperm with non-linear motility: Forward movement but with a tendency toward \\
& curved or crooked trajectories \\
Grade 4 & Sperm with progressive motility: Strong, fast swimming in a straight line \\
\hline
\end{tabular}


determined with an ELISA method (Cayman's 8-Isoprostane EIA Kit, No. 516351, Cayman Chemical Company, Ann Arbor, MI, USA). Intra- and interassay variation was $12.6-19.9 \%$ and $10.5-9.6 \%$, respectively.

All experimental protocols were used under approval from the Animal Care and Use Committee for Animal Investigation. The experimental procedures were conducted in a manner consistent with the relevant ethical guidelines for animal research.

\section{STATISTICAL ANALYSIS}

The distribution of variables was studied with probability plots and the Shapiro-Wilks test. The post-intervention differences between groups were analyzed by one-way analysis of variance (ANOVA). The least significant difference (LSD) test was used wherever there was a main effect to compare variables between each group separately; $p<0.05$ was accepted as significant.

Statistical analyses were done with the SPSS v. 15 statistical software package (SPSS Inc., Chicago, IL, USA).

\section{RESULTS}

\section{Body Weight}

Mean body weight in all four groups during the 86-day study period is shown in Table 2. At baseline, mean body weight was $115.41 \pm 15.59 \mathrm{~g}$ in group $1,115.66 \pm$ $18.08 \mathrm{~g}$ in group 2, $115.50 \pm 14.73 \mathrm{~g}$ in group 3 , and $115.25 \pm 17.06 \mathrm{~g}$ in group 4; and at the end of the study, body weight had increased to $284.41 \pm 45.95 \mathrm{~g}, 258.50 \pm$ $13.39 \mathrm{~g}, 267.91 \pm 47.77 \mathrm{~g}$, and $232.16 \pm 23.18 \mathrm{~g}$, respectively. The changes in weight in group 2 was significantly lower than in group 1 , and it was also significantly lower in group 4 than in group 3 (Table 2).

\section{Total Calorie Intake}

Total calorie intake during the 86-day study period in group 2 was significantly lower than in group 1, and it was also significantly lower in group 4 than in group 3 (Table 2).

\section{Epididymis Weight}

Epididymis weight and epididymis fat/epididymis ratio did not differ significantly between groups at the end of the study period (Table 2).

\section{Sperm Count and Motility}

After 86 days, a significant increase in mean sperm count was seen in group 4 $(p=0.01)$ compared to group 3 (Table 2$)$. Percentage of immotile sperm was significantly lower in groups 2,3 , and 4 compared to group $1(p=0.001)$.

\section{Serum Antioxidants}

A significant increase in TAC was observed in group $3(p=0.02)$ and group 4 $(p=0.001)$ compared to groups 1 and 2 (Table 2$)$. In rats given dietary antioxidant 


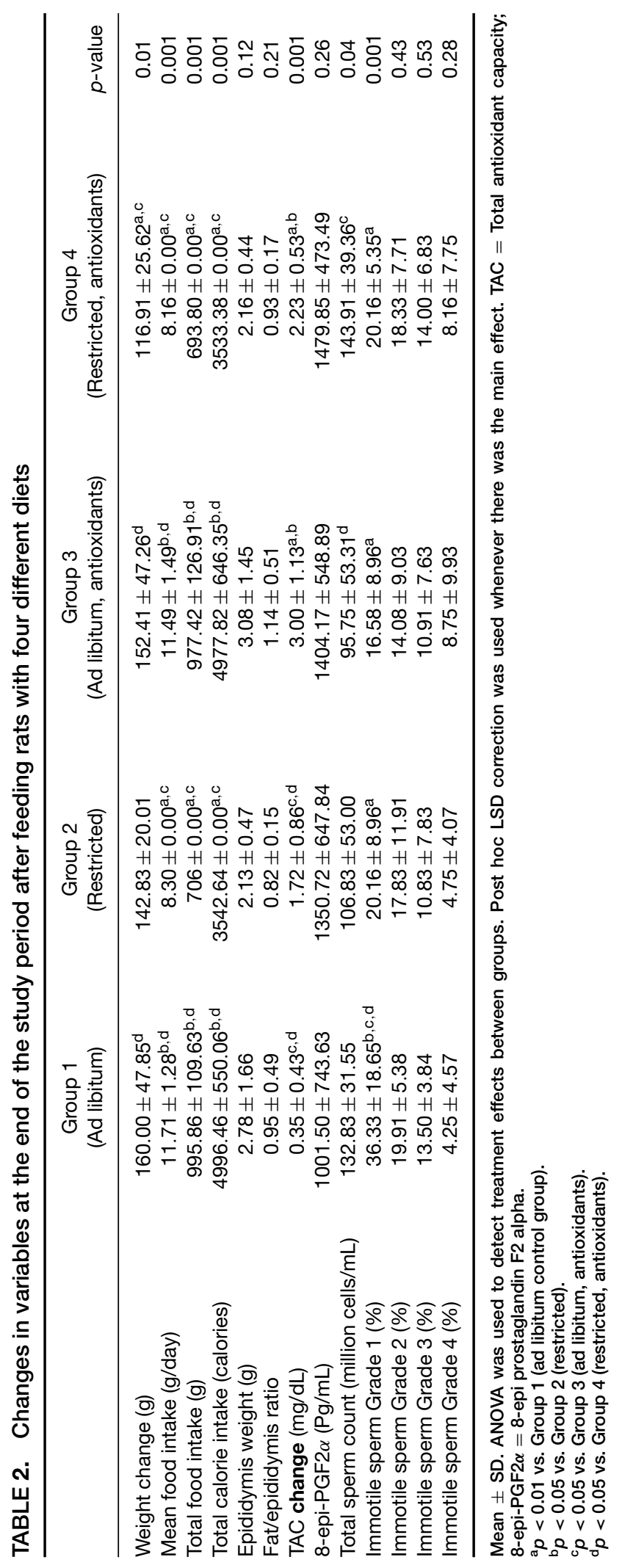


supplementation with (group 4) or without calorie restriction (group 3), there were no significant differences in 8-epi-PGF2 $\alpha$ compared to groups 1 or 2 (Table 2).

\section{DISCUSSION}

The findings of this study showed a significant increase in sperm count and quality in the groups of rats fed with a mixture of astaxanthin, vitamin E, and vitamin $\mathrm{C}$ combined with calorie restriction. Some studies have reported increased fertility after weight loss (Heracek, Sobotka, \& Urban, 2012; Katib, 2015). Moderate weight loss during long-term calorie restriction is associated with a marked clinical improvement in insulin resistance (Kiddy et al., 1992). Low testosterone levels are known to be correlated with insulin resistance and obesity (Tsai et al., 2004). The low sperm counts observed in male obesity are, at least in part, a result of changes in the hypothalamus-pituitary gland (HPG) axis through decreased testosterone levels (Grossmann, 2011). Our data, however, did not show any significant change in sperm count after feeding with a calorie-restricted diet.

A paradoxical impact of calorie restriction on infertility has been reported (Black et al., 2001; Lane, Ingram, \& Roth, 1997; Lane et al., 2001; Roth et al., 2000; Sitzmann et al., 2014). Data from studies in female rhesus macaques show that reproductive hormones are not affected by long-term moderate calorie restriction, whereas in male rhesus macaques, calorie restriction inhibits maturation-related testosterone secretion (Black et al., 2001; Lane et al., 1997; Roth et al., 2000).

The administration of astaxanthin, combined with vitamin $\mathrm{E}$ and vitamin $\mathrm{C}$, in our study appeared to play an essential role in improving sperm quality and quantity in rats. Kessopoulou et al. (1995) reported that the oral administration of vitamin E significantly improved the in vitro function of human spermatozoa as assessed by the Zona binding test. Vitamin $\mathrm{C}$ also has been shown to enhance sperm quantity and improve semen quality in rats (Vijayprasad et al., 2014).

Recently, astaxanthin has been reported to improve sperm capacitation and functions by improving redox system imbalance (Tripathi \& Jena, 2008). Comhaire and colleagues (2003) found that astaxanthin decreased ROS produced by Sertoli cells. In addition, astaxanthin reportedly protects steroidogenesis from hydrogen peroxide-induced oxidative stress in mouse Leydig cells (Wang et al., 2015). It should be noted that testosterone as a steroid is always at risk of oxidation.

The relationship between leptin, antioxidants, and male infertility may provide an explanation for improved sperm counts after dietary antioxidant supplementation. A strong link was found between adipocyte secretion of leptin and gonadotropin hormone secretion. Since leptin regulates gonadotropin-releasing hormone secretion in the hypothalamus, it acts as a surreptitious signal that associates metabolic status to the reproductive axis (Hausman, Barb, \& Lents, 2012). An increase in leptin levels significantly decreases the production of testosterone from Leydig cells (Caprio et al., 1999). This effect explains why high leptin levels, usually found in obese males (Caro et al., 1996), might affect the HPG axis and lead to decreased testosterone production. Several reports found that antioxidants also decrease leptin levels. For example, Xiong et al. (2014) reported that vitamin E decreased serum leptin by reducing the signal transducer and activator of 
transcription 3. In addition, vitamin C causes a dramatic decrease in leptin secretion by inhibiting insulin receptor substrate 3 (irs3) (Garcia-Diaz et al., 2010).

Reportedly, astaxanthin is also able to reduce leptin levels. Ruscica et al. (2014) administered astaxanthin to 30 patients with metabolic syndrome and observed improvements in the leptin to adiponectin ratio. Together, these studies and our results suggest that a synergic impact of calorie restriction and antioxidant supplementation on leptin secretion enhances sperm count and motility, and may thus improve fertility.

Although the calorie-restricted diet used in the present study did not change sperm count, it increased sperm motility compared with the rats fed with ad libitum diet. This is consistent with the findings published by Fernandez et al. (2011), who reported that sperm quality was reduced in rats fed with a diet intended to induce fattening, i.e., the percentage of sperm with progressive movement decreased. Kort et al. (2006) also found an inverse relationship between body mass index (BMI) and the total number of normal-motile sperm cells. A study in male New Zealand white rabbits that were made diabetic found high levels of advanced glycation end product $N \varepsilon$-carboxymethyl-lysine and its receptor in the testes, epididymis, and sperms (Mallidis et al., 2011). Glycation end products are known to cause cellular dysfunction, oxidative stress, and deoxyribonucleic acid (DNA) damage in various organs under a variety of conditions (Vlassara \& Palace, 2002). We hypothesize that they may also play a role in reduced sperm motility.

Calorie restriction is known to improve insulin resistance and reduce the amount of advanced glycation end products (Aris et al., 2010). In this connection, we found that dietary supplementation with astaxanthin combined with vitamin $\mathrm{E}$ and vitamin $\mathrm{C}$ also decreased the percentage of non-motile sperms regardless of whether the animals were allowed food ad libitum or given a calorie-restricted diet. This result is consistent with the study by ElSheikh et al. (2015), who found improvements in mean total sperm motility after six months of vitamin E supplementation. Zhao et al. (2015) also examined the effect of a mixture of vitamin $\mathrm{E}$ and vitamin $\mathrm{C}$ on sperm motility in bulls, and observed the same result. Moreover, dietary supplementation with astaxanthin has been found to improve sperm motility in both animal models and humans (Comhaire \& Mahmoud, 2003; Tizkar et al., 2015). Collectively, ROS damage cytoplasmic organelles, such as the sperm flagellum, and thus lead to loss of motility (Aitken, Jones, \& Robertson, 2012). Our results suggest that antioxidant agents such as astaxantin, vitamin $\mathrm{E}$, and vitamin $\mathrm{C}$ may improve sperm motility by inhibiting ROS production.

We observed no significant changes in epididymis weight or fat/epididymis ratio in the present study. This result is consistent with the findings of Edmonds, Dallie, and Withyachumnarnkul (1982), who reported no difference in the weight of testes or ventral prostate in Zucker rats, a genetic model of obesity, compared with lean rats. Similarly, diet-induced obesity in male mice was not associated with changes in the average weight of testes or epididymis (Ghanayem et al., 2010). These findings show that the direct influence of calorie restriction and antioxidant supplementation (together or separately) on ROS and consequently spermatogenesis or sperm quality is independent of total fat content in the epididymis. 


\section{LIMITATIONS AND STRENGTHS OF THE STUDY}

The main strength of this study was that we used more than one antioxidant to regenerate astaxanthin, a major advantage compared to previous studies that tested only one antioxidant.

The main limitation of our study was that we did not measure sperm quality and quantity, or determine epididymis weight or the fat/epididymis ratio at the beginning of the study - this would have made it possible to examine changes in these parameters during the study period. However, it is not possible to weigh the epididymis or determine the fat/epididymis ratio without killing the rats.

\section{CONCLUSIONS}

Feeding astaxanthin in combination with vitamin $\mathrm{E}$ and vitamin $\mathrm{C}$, together with calorie restriction, was able to ameliorate, at least in part, infertility in male rats. It is noteworthy to mention that life style modifications (calorie restriction and antioxidant consumption) lead to improvement in fertility potency.

\section{ACKNOWLEDGMENTS}

This study was supported in part by a grant from The Hamedan University of Medical Sciences, Hamedan, I.R. Iran (DP/20/ 18/3/1237, 8/9/2012). We thank K. Shashok (author AID in the Eastern Mediterranean) for improving the use of English in the manuscript. We performed this research only in the interest of knowledge.

Declaration of Interest: The authors report no conflicts of interest. The authors alone are responsible for the content and writing of the article.

\section{ABOUT THE AUTHORS}

Aliasghar Vahidinia, Department of Nutrition, School of Medicine, Hamadan University of Medical Sciences, Hamadan, I.R. Iran. Ali Reza Rahbar, Department of Nutrition, The Persian Gulf Tropical Medicine Research Center, Bushehr University of Medical Sciences, Bushehr, I.R. Iran. Mohammad Mehdi Shakoori Mahmoodabadi, Department of Nutrition, School of Medicine, Hamadan University of Medical Sciences, Hamadan, I.R. Iran.

\section{REFERENCES}

Agarwal A, Saleh RA. Role of oxidants in male infertility: rationale, significance, and treatment. Urol Clin North Am. 2002;29:817-827.

Agarwal A, Tvrda E, Sharma R. Relationship amongst teratozoospermia, seminal oxidative stress and male infertility. Reprod Biol Endocrinol. 2014;12:45.

Aitken RJ, Jones KT, Robertson SA. Reactive oxygen species and sperm function - in sickness and in health. J Androl. 2012;33:1096-1106.

Aitken RJ, Roman SD. Antioxidant systems and oxidative stress in the testes. Oxid Med Cell Longev. 2008;1:15-24. 
Andrisani A, Dona G, Tibaldi E, Brunati AM, Sabbadin C, Armanini D, et al. Astaxanthin improves human sperm capacitation by inducing Lyn displacement and activation. Marine Drugs. 2015;13:5533-5551.

Aris JP, Elios MC, Bimstein E, Wallet SM, Cha S, et al. Gingival RAGE expression in calorierestricted versus ad libitum-fed rats. J Periodont. 2010;81:1481-1487.

Black A, Allison DB, Shapses SA, Tilmont EM, Handy AM, et al. Calorie restriction and skeletal mass in rhesus monkeys (Macacamulatta): evidence for an effect mediated through changes in body size. J Gerontol A. 2001;56:B98-B107.

Caprio M, Isidori AM, Carta AR, Moretti C, Dufau ML, Fabbri A. Expression of functional leptin receptors in rodent Leydig cells. Endocrinology. 1999;140:4939-4947.

Caro JF, Kolaczynski JW, Nyce MR, Ohannesian JP, OpentanovaI, et al. Decreased cerebrospinal-fluid/serum leptin ratio in obesity: a possible mechanism for leptin resistance. Lancet (London, England). 1996;348:159-161.

Comhaire FH, Mahmoud A. The role of food supplements in the treatment of the infertile man. Reprod Biomed Online. 2003;7:385-391.

du Plessis SS, Kashou A, Vaamonde D, Agarwal A. Is there a link between exercise and male factor infertility. Open Reprod Sci J. 2011;3:105-113.

Edmonds ES, Dallie SK, Withyachumnarnkul B. Reproductive system of the obese male zucker rat. Reproductive capacity, artificial insemination and plasma testosterone levels. Biol.Reprod. 1982;27:891-897.

El Sheikh MG, Hosny MB, Elshenoufy A, Elghamrawi H, Fayad A, Abdelrahman S. Combination of vitamin $\mathrm{E}$ and clomiphene citrate in treating patients with idiopathic oligoasthenozoospermia: a prospective, randomized trial. Andrology. 2015;3:864-867.

Erel O. A novel automated direct measurement method for total antioxidant capacity using a new generation, more stable ABTS radical cation. Clin Biochem. 2004;37:277-285.

Fernandez CD, Bellentani FF, Fernandes GS, Perobelli JE, Favareto AP, et al. Diet-induced obesity in rats leads to a decrease in sperm motility. Reprod Biol Endocrinol. 2011;9:32.

Garcia-Diaz DF, Campion J, Milagro FI, Boque N, Moreno-Aliaga MJ, Martinez JA. Vitamin C inhibits leptin secretion and some glucose/lipid metabolic pathways in primary rat adipocytes. JMol Endocrinol. 2010;45:33-43.

Ghanayem BI, Bai R, Kissling GE, Travlos G, Hoffler U. Diet-induced obesity in male mice is associated with reduced fertility and potentiation of acrylamide-induced reproductive toxicity. BiolReprod. 2010;82:96-104.

Grossmann M. Low testosterone in men with type 2 diabetes: significance and treatment. J Clin Endocrinol Metab. 2011;96:2341-2353.

Guerin M, Huntley ME, Olaizola M. Haematococcusastaxanthin: applications for human health and nutrition. Trends Biotechnol. 2003;21:210-216.

Gvozdjakova A, Kucharska J, Dubravicky J, Mojto V, Singh RB. Coenzyme Q (10), alphatocopherol, and oxidative stress could be important metabolic biomarkers of male infertility. Dis Markers. 2015:827941.

Hausman GJ, Barb CR, Lents CA. Leptin and reproductive function. Biochimie. 2012;94:2075-2081.

Heracek J, Sobotka V, Urban M. Obesity and male infertility. Ceska Gynekol. 2012;77:450-456.

Jensen TK, Andersson AM, Jorgensen N, Andersen AG, Carlsen E, et al. Body mass index in relation to semen quality and reproductive hormones among 1558 Danish men. Fertil Steril. 2004;82:863-870.

Katib A. Mechanisms linking obesity to male infertility. Cent Eur J Urol. 2015;68:79-85.

Kessopoulou E, Powers HJ, Sharma KK, Pearson MJ, Russell JM, et al. A double-blind randomized placebo cross-over controlled trial using the antioxidant vitamin $\mathrm{E}$ to treat reactive oxygen species associated male infertility. Fertil Steril. 1995;64:825-831.

Kiddy DS, Hamilton-Fairley D, Bush A, Short F, Anyaoku V, et al. Improvement in endocrine and ovarian function during dietary treatment of obese women with polycystic ovary syndrome. Clin Endocrinol. 1992;36:105-111.

Kort HI, Massey JB, Elsner CW, Mitchell-Leef D, Shapiro DB, et al. Impact of body mass index values on sperm quantity and quality. J Androl. 2006;27:450-452. 
Lane MA, Black A, Handy AM, Shapses SA, Tilmont EM, et al. Energy restriction does not alter bone mineral metabolism or reproductive cycling and hormones in female rhesus monkeys. $\mathbf{J}$ Nutr. 2001;131:820-827.

Lane MA, Ingram DK, Roth GS. Beyond the rodent model: calorie restriction in rhesus monkeys. Age (Omaha). 1997;20:45-56.

Mallidis C, Czerwiec A, Filippi S, O'Neill J, Maggi M, McClure N. Spermatogenic and sperm quality differences in an experimental model of metabolic syndrome and hypogonadalhypogonadism. Reproduction (Cambridge, England). 2011;142:63-71.

Nagata C, Takatsuka N, Kawakami N, Shimizu H. Relationships between types of fat consumed and serum estrogen and androgen concentrations in Japanese men. Nutr Cancer. 2000;38:163-167.

Pashkow FJ, Watumull DG, Campbell CL. Astaxanthin: a novel potential treatment for oxidative stress and inflammation in cardiovascular disease. Am J Cardiol. 2008;101:58D-68D.

Palmer NO, Bakos HW, Fullston T, Lane M. Impact of obesity on male fertility, sperm function and molecular composition. Spermatogenesis. 2012;2:253-263.

Rathje TA, Johnson R, Lunstra D. Sperm production in boars after nine generations of selectionfor increased weight of testis. J Anim Sci.. 1995;73:2177-2185.

Roth GS, Ingram DK, Black A, Lane MA. Effects of reduced energy intake on the biology of aging: the primate model. Eur J Clin Nutr. 2000;3(54 Suppl):S15-S20.

Ruscica M, Gomaraschi M, Mombelli G, Macchi C, Bosisio R, et al. Nutraceutical approach to moderate cardiometabolic risk: results of a randomized, double-blind and crossover study with Armolipid Plus. J Clin Lipid. 2014;8:61-68.

Sallmen M, Sandler DP, Hoppin JA, Blair A, Baird DD. Reduced fertility among overweight and obese men. Epidemiology. 2006;17:520-523.

Sigurgisladottir S, Parrish C, Lall S, Ackman R. Effects of feeding natural tocopherols and astaxanthin on Atlantic salmon (Salmosalar) fillet quality. Food Res Int. 1994;27:23-32.

Sitzmann BD, Brown DI, Garyfallou VT, Kohama SG, Mattison JA, et al. Impact of moderate calorie restriction on testicular morphology and endocrine function in adult rhesus macaques (Macacamulatta). Age (Dordr). 2014;36:183-197.

Stephen EH, Chandra A. Declining estimates of infertility in the United States: 1982-2002. Fertil Steril. 2006;86:516-523.

Tizkar B, Kazemi R, Alipour A, Seidavi A, Naseralavi G, Ponce-Palafox JT. Effects of dietary supplementation with astaxanthin and beta-carotene on the semen quality of goldfish (Carassiusauratus). Theriogenology. 2015;84:1111-1117.

Tripathi DN, Jena GB. Astaxanthin inhibits cytotoxic and genotoxic effects of cyclophosphamide in mice germ cells. Toxicology. 2008;248:96-103.

Tsai EC, Matsumoto AM, Fujimoto WY, Boyko EJ. Association of bioavailable, free, and total testosterone with insulin resistance: influence of sex hormone-binding globulin and body fat. Diabetes Care. 2004;27:861-868.

Vasantha Rupasinghe HP, Yasmin A. Inhibition of oxidation of aqueous emulsions of omega3 fatty acids and fish oil by phloretin and phloridzin. Molecules (Basel, Switzerland). 2010;15:251-257.

Vijayprasad S, Ghongane B, Nayak B. Effect of vitamin C on male fertility in rats subjected to forced swimming stress. J Clin Diagn Res. 2014;8:HC05.

Vincent HK, Bourguignon CM, Vincent KR, Weltman AL, Bryant M, Taylor AG. Antioxidant supplementation lowers exercise-induced oxidative stress in young overweight adults. Obesity (Silver Spring, MD). 2006;14:2224-2235.

Vlassara H, Palace MR. Diabetes and advanced glycationend products. J Intern Med. 2002;251:87-101.

Wang JY, Lee YJ, Chou MC, Chang R, Chiu CH, et al. Astaxanthin protects steroidogenesis from hydrogen peroxide-induced oxidative stress in mouse Leydig cells. Marine Drugs. 2015;13:1375-1388.

Wolf AM, Asoh S, Hiranuma H, Ohsawa I, Iio K, et al. Astaxanthin protects mitochondrial redox state and functional integrity against oxidative stress. J Nutr Biochem. 2010;21:381389. 
Xiong RB, Li Q, Wan WR, Guo JQ, Luo BD, Gan L. Effects and mechanisms of vitamin A and vitamin $\mathrm{E}$ on the levels of serum leptin and other related cytokines in rats with rheumatoid arthritis. Exp Therap Med. 2014;8:499-504.

Zhang P, Omaye S. Antioxidant and prooxidant roles for $\beta$-carotene, $\alpha$-tocopherol and ascorbic acid in human lung cells. Toxicol Vitro. 2001;15:13-24.

Zhao XL, Li YK, Cao SJ, Hu JH, Wang WH, et al. Protective effects of ascorbic acid and vitamin E on antioxidant enzyme activity of freeze-thawed semen of Qinchuan bulls. Genet Mol Res. 2015;14:2572-2581.

Zorn B, Vidmar G, Meden-Vrtovec H. Seminal reactive oxygen species as predictors of fertilization, embryo quality and pregnancy rates after conventional in vitro fertilization and intracytoplasmic sperm injection. Int J Androl. 2003;26:279-285. 